\title{
Commissioning of the ATLAS Tile Calorimeter with Cosmic Ray and Single Beam Data
}

\author{
Hideki Okawa, Student Member, IEEE, on behalf of the ATLAS Collaboration
}

\begin{abstract}
The commissioning of the ATLAS detector at the Large Hadron Collider (LHC) has been the focus of an extensive program over several years. We present a summary of cosmic ray and single beam commissioning results with the ATLAS hadronic Tile Calorimeter (TileCal). The emphasis will be on understanding the energy and timing reconstruction, and validation of their calibration. Finally, electronic noise at the cell level is studied, and performance of calorimeter clusters is investigated.
\end{abstract}

\section{INTRODUCTION}

$\mathbf{T}$ HE ATLAS detector (Fig. 1) is a general-purpose detector placed at Point 1 of the 4 interaction regions at the Large Hadron Collider (LHC) [1] which protons will collide at the center-of-mass energy of $14 \mathrm{TeV}$ with the peak design luminosity of $10^{34} \mathrm{~cm}^{-2} \mathrm{~s}^{-1}$. It is designed to meet requirements from various physics measurements such as searches for Higgs particles and physics beyond the Standard Model.

\section{A. The ATLAS Detector}

§ิ The ATLAS detector [2] consists of inner detectors, an ó electromagnetic (EM) calorimeter, a hadronic calorimeter,

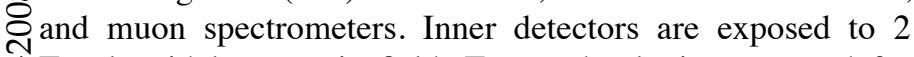
Ú T solenoidal magnetic field. Two technologies are used for calorimeters; liquid argon and scintillating tiles. The liquid regon technique is used for the EM calorimeter, end-cap 1adronic calorimeter, and forward calorimeter. Scintillating $\cup$ tides are used for the barrel hadronic calorimeter. The calorimeII tris achieve unprecedented granularity and coverage up to F pseudorapidity $(\eta)$ 5.0. Three toroidal magnets are used for 当尚uon spectrometers. ATLAS exploits many new technologies $\varangle$ stich as hybrid hadronic calorimeter technique (two different ztechniques are used in the barrel and end-cap) and air-core 递地oidal magnets for muon track measurement, to name a few.

\section{B. Tile Calorimeter}

Tile Calorimeter (TileCal) [3] (Fig. 2) is a hadronic sampling calorimeter in the barrel region which consists of 3 cylindrical sections. The Long-Barrel (LB: $|\eta|<1.0$ ) is contained in a single cylinder with separate partitions for positive and negative $\eta$. Two partitions of the Extended-Barrel (EB: $0.8<|\eta|<1.7)$ are respectively contained in a cylinder. The 4 partitions are named as LBA, LBC, EBA, and $\mathrm{EBC}$, where A corresponds to the positive $\eta$ (Anti-clockwise direction of the beam-line) and $\mathrm{C}$ the negative $\eta$ (Clockwise direction). TileCal

Manuscript received November 14, 2008

H. Okawa is with the Department of Physics, the University of Tokyo, Japan (e-mail: okawa@icepp.s.u-tokyo.ac.jp) and a JSPS (Japan Society for the Promotion of Science) Research Fellow.

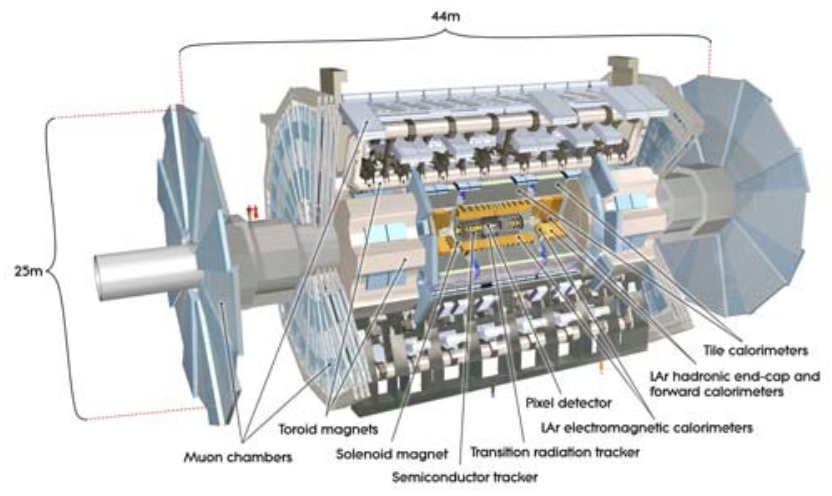

Fig. 1. The ATLAS Detector.

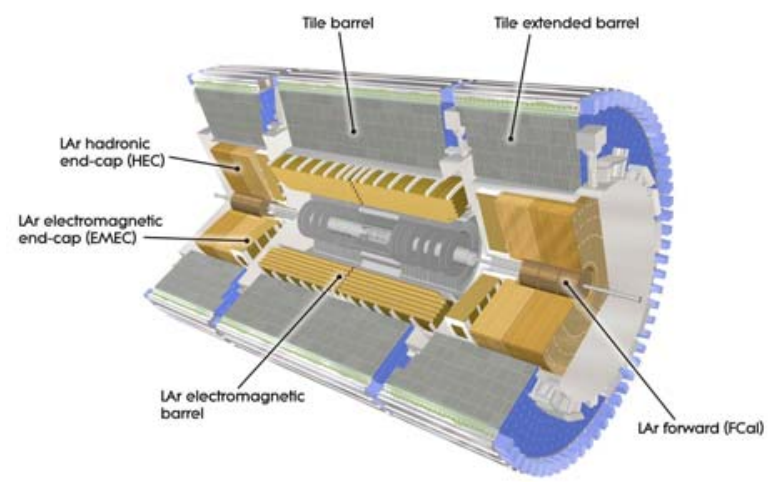

Fig. 2. ATLAS Calorimeters. They consist of Liquid Argon (LAr) EM calorimeter, Tile barrel hadronic calorimeter, LAr end-cap hadronic calorimeter, and LAr forward calorimeter.

has 3 sampling layers (sampling A, BC, D). Scintillating tiles are used as active material and steel is used as an absorber. Tiles are placed perpendicular to the beam axis and radially staggered in depth, which is a new technology and enables simpler readout and tile configuration [4] (Fig. 3). Signals are read out by photomultipliers (PMTs) with wavelength shifting (WLS) fibers connected to both side of the tiles. TileCal has good time resolution, around $1 \mathrm{~ns}$, and adequate granularity $(\triangle \eta \times \triangle \phi=0.1 \times 0.1$, and $0.2 \times 0.1$ for the last layer) to achieve good enough jet energy and missing transverse energy resolution.

\section{CAlibration in Tile Calorimeter}

For accurate reconstruction of energy of physics objects, TileCal has sophisticated calibration systems to take care of all the signal paths in the calorimeter (Fig. 4). The systems consist of a movable Cesium $\left({ }^{137} \mathrm{Cs}\right)$ radioactive source for 


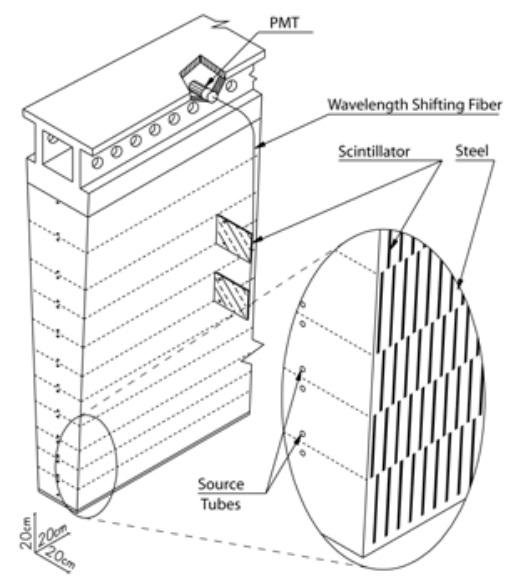

Fig. 3. Schematic view of the mechanical assembly and the optical readout of the Tile Calorimeter.

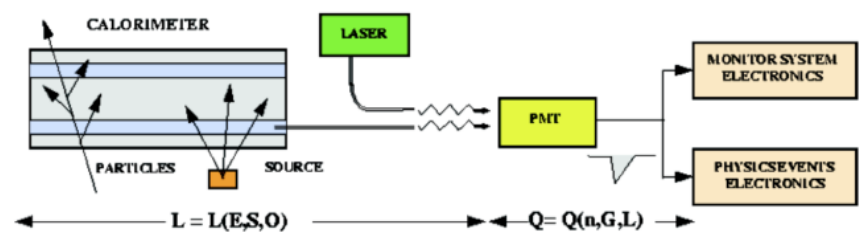

Fig. 4. Calibration scheme of the ATLAS Tile Calorimeter; L(E,S,O) is light response $(\mathrm{E}=$ energy, $\mathrm{S}=$ sampling fraction, $\mathrm{O}$ : response of optical component (scintillator and WLS fibers)). Light pulse is converted into a charge $Q(n, G, L)$ at PMTs ( $n=$ quantum efficiency, $\mathrm{G}=$ gain of the PMT).

monitoring the scintillator and PMT response, a Minimum Bias (MB) monitoring system which will use Minimum Bias events to monitor the calorimeter response during physics runs, the pulsed laser system for monitoring the PMT and readout response, and the electronic Charge Injection System (CIS) solely for monitoring the readout. TileCal have a dedicated readout (monitor system electronics) for the $\mathrm{Cs}$ and $\mathrm{MB}$ monitoring systems in addition to the fast readout used for the laser, CIS calibration systems, and for events from the physics runs.

The Cs scans are done outside the beam periods. They enable the cell intercalibration to equalize the signal response from all the cells, and also to monitor the cell response in time. The EM scale factor $(\mathrm{pCb} / \mathrm{GeV})$ is defined from testbeam analyses with $20 \%$ of the whole modules, and the same factor is applied to all the cells. Online cell intercalibration is done by adjusting the high voltage (HV) of the PMTs based on a fast analysis of the Cs scanned data. An additional offline cell correction is applied using a more refine calibration method.

Laser runs will be accompanying Cs scans and will also be taken in between physics runs to understand PMT response changes in regards to linearity and gains. The laser system can measure the absolute gains of each PMT as well, and is also used to calibrate in time the readout digitizers.

In between physics runs, pedestal, laser, and CIS runs will be taken. The CIS is a system which injects well defined charge into fast bi-gain electronics. This system provides $\mathrm{ADC} / \mathrm{pC}$ conversion for both gains. It also provides an offline correction for nonlinearity in the low-gain.

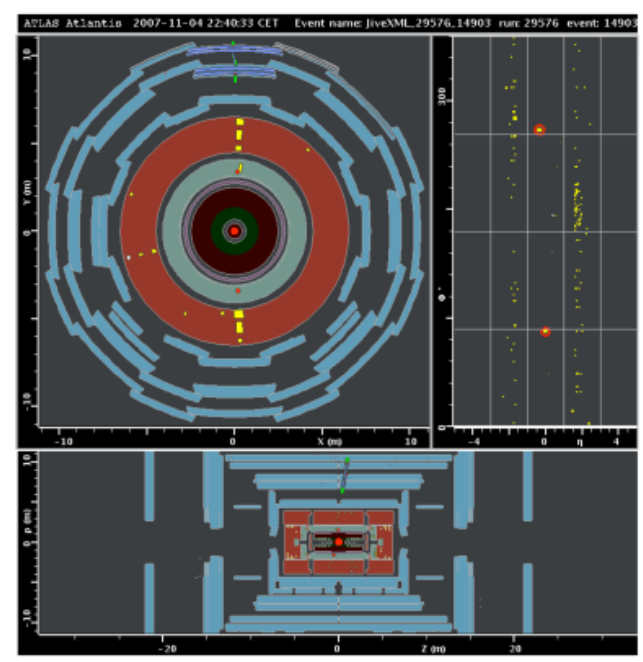

Fig. 5. Cosmic muon event observed with the ATLAS detector in Nov. 2007.

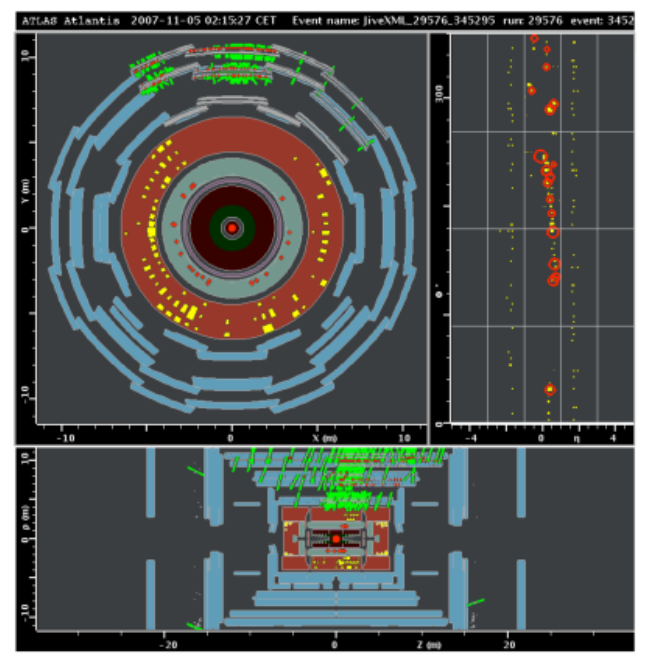

Fig. 6. Cosmic shower event observed with the ATLAS detector in Nov. 2007.

\section{COMMISSIONING RUNS}

\section{A. Cosmic Ray Data Taking}

Cosmic ray commissioning has started in June 21, 2005. TileCal was the first sub-detector to be included in ATLAS, and took the "first data" in the LHC [5]. There have been a number of dedicated combined cosmic runs with many subdetectors included since December 2006 (so called "Milestone Weeks" [6]). Many cosmic events (Fig. 5) and some shower events (Fig. 6) are recorded up to now, which helped to understand the detectors and validate calibrations.

\section{B. Single Beam Data Taking}

The first circulations of single beams were achieved on September 10 to 12,2008 . Circulations of beams in both directions were successfully carried out. Many beam halo muons and other particles originated from beams interacting with the collimator 148 meter upstream from the ATLAS detector, and were observed in TileCal and other sub-detectors (Fig. 7). 


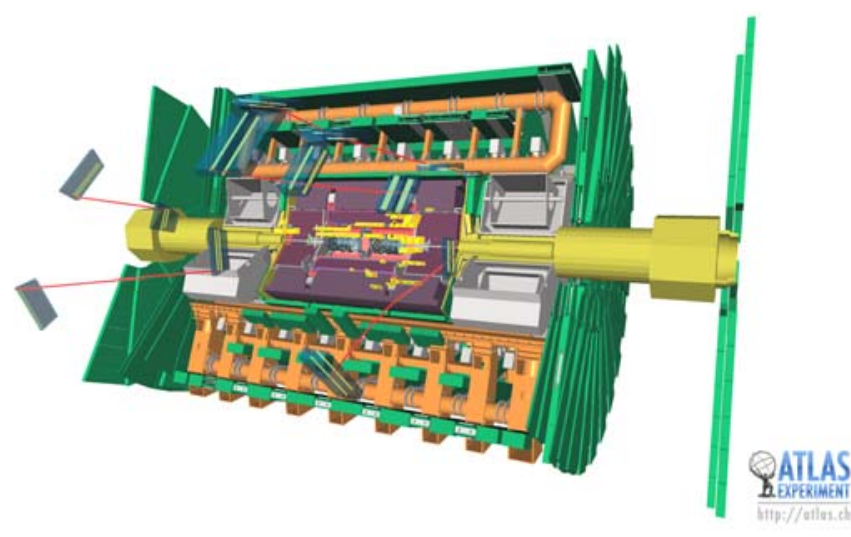

Fig. 7. 3D event display of a beam halo event observed in Sep. 2008.

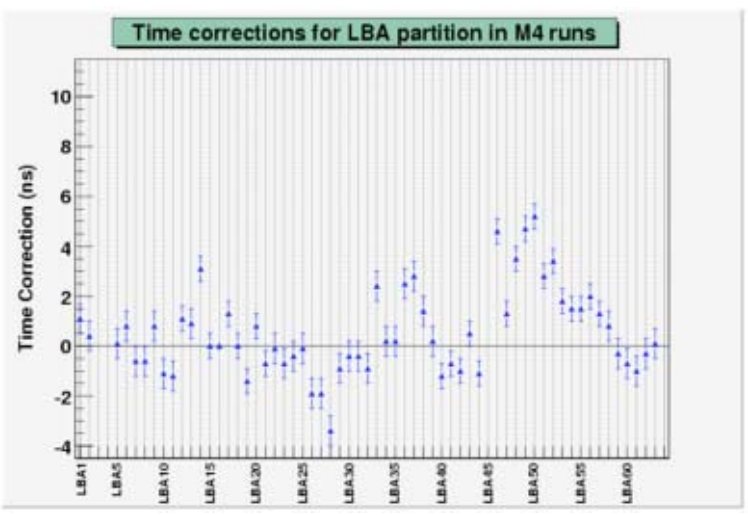

Fig. 8. Time corrections for LBA modules obtained from combined cosmic runs in Sep. 2007.

Beam halo muons provide additional information to that from cosmic muons, especially on inter-partition information.

\section{Tile Calorimeter Commissioning With Cosmic AND SINGLE BEAM DATA}

Cosmic and single beam data enable the first in-situ measurement with physics particles before the collisions. Energy deposits from cosmic or beam halo muons provide useful information to validate energy and timing calibration.

These commissioning runs also enable the first studies on the performance of calorimeter clusters in-situ.

\section{A. Validation of Timing Calibration}

To validate the timing calibration obtained from the laser calibration system, the time-of-flight of cosmic and beam halo muons was used.

In cosmic ray data, events triggered by Resistive-Plate Chambers (RPC) which is a barrel muon trigger, were used [7]. A module (LBA16) in the top drawer was fixed as the reference, and time-of-flight between cells in LBA16 and other drawers was compared with the expected time calculated by considering the distance between the two cells. A good agreement with the laser calibration was observed (Fig. 8). After this timing correction method, the time resolution will reach less than $0.7 \mathrm{~ns}$.

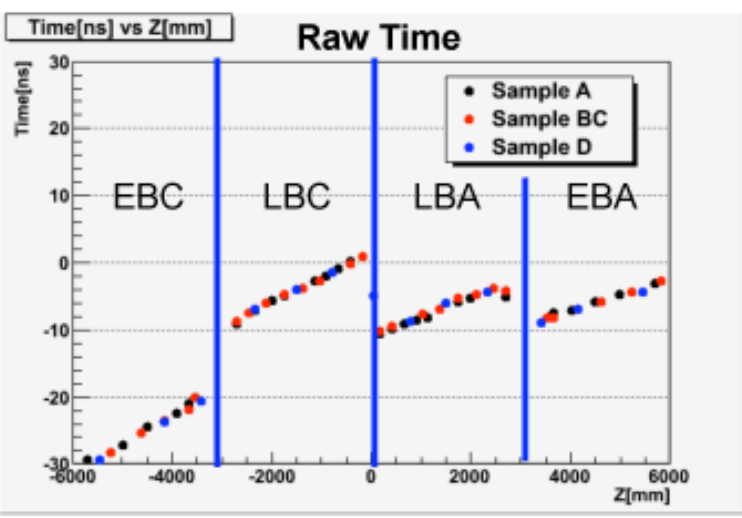

Fig. 9. Timing information as a function of $\mathrm{Z}$ (beam direction), obtained from single beam runs.

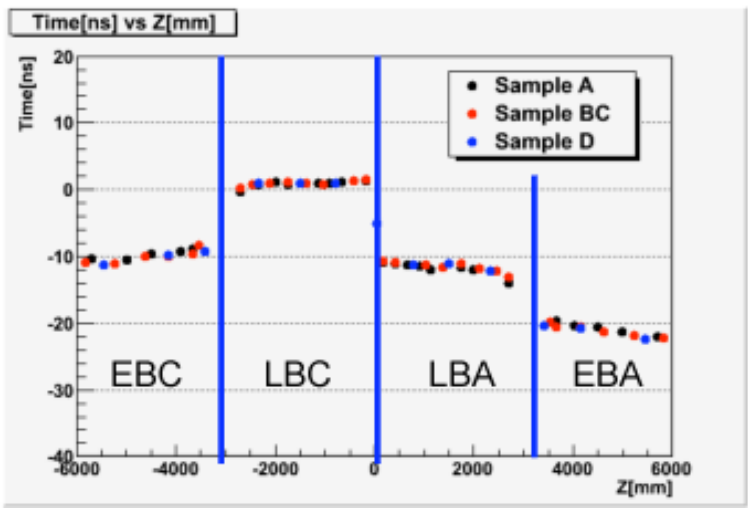

Fig. 10. Timing information as a function of $\mathrm{Z}$ after time-of-flight correction from single beam runs.

In single beam data, the signal timing was investigated as a function of $\mathrm{Z}$ (beam direction), and the time-of-flight was calculated in the lateral direction. The laser corrections which take into account the laser and WLS fiber lengths were applied for the timing calculation. In addition to the corrections, one more correction was applied which considers the fact that beam halo particles were not coming from the interaction point (IP), but traveling along the beam direction. In Fig. 9, the time as a function of $\mathrm{Z}$ position is shown. The slope is consistent with the fact that the particles are traveling from the negative $\eta$ side to the positive side. After applying the time-of-flight correction on each cell (Fig. 10), flat response of the timing with a dispersion within $2 \mathrm{~ns}$ was observed in the same partition, which confirms that the timing calibration was in good agreement. However, a slight residual slope still suggests some improvements in the calibration. A timing discontinuity was observed among the partitions as well, though the differences of the offsets were within 1 bunch crossing (25 ns). These effects are to be improved, and are under investigation.

\section{B. Validation of Energy Calibration}

As time-of-flight of cosmic and beam halo muons was useful references for validating the timing calibration, energy loss of those particles in TileCal enables the validation of the energy calibration. 


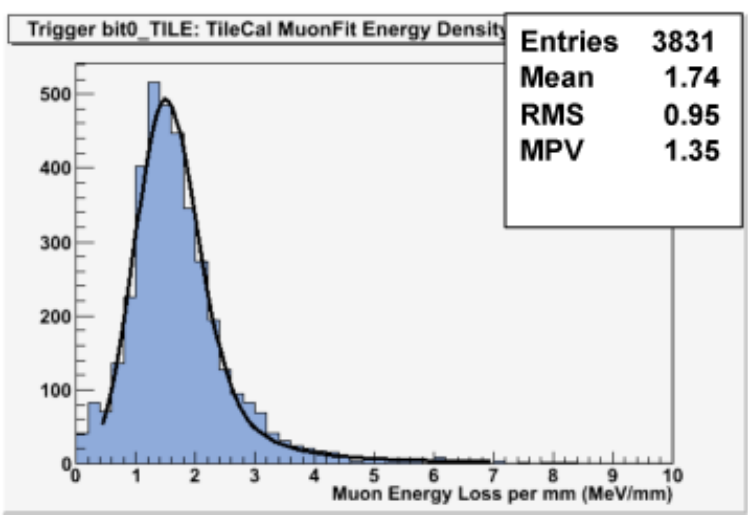

Fig. 11. Muon energy loss from cosmic runs.

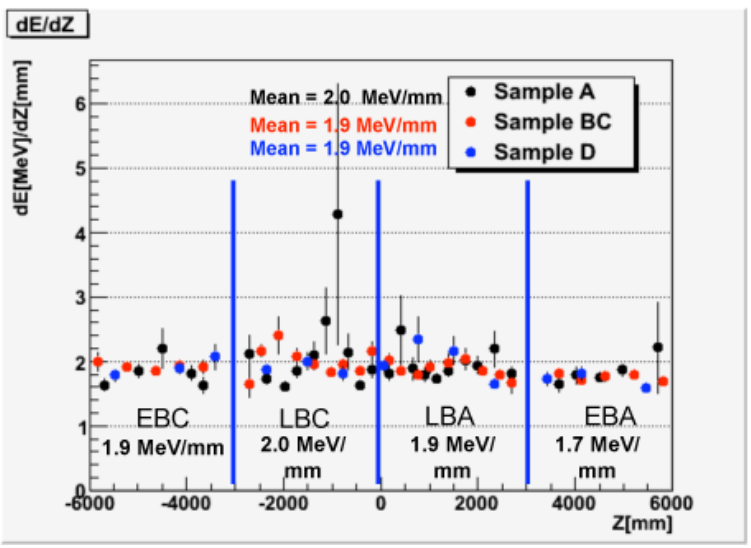

Fig. 12. Mean muon energy loss from single beam runs as a function of the beam direction.

The energy loss of cosmic muons is shown in Fig. 11, where muons were identified with the "TileMuonFitter" algorithm [8] which reconstructs muons in TileCal. The mean energy loss was calculated from the sum of the energy of cells within particular radius (it depends on the sampling layer) from the muon track divided by the path length. Energy loss was consistent with what we expect from the muons in TileCal.

The energy loss in the beam direction was measured from the single beam runs as well (Fig. 12). Energy was measured cell by cell, summed in $\phi$ direction and was normalized by the average number of tiles. After considering that 1 period of tile is $18 \mathrm{~mm}$, and divide the value by the number of observed muons, mean energy loss in the beam direction $(\mathrm{dE} / \mathrm{dZ}[\mathrm{MeV} / \mathrm{mm}])$ was obtained. Energy loss is consistent between cosmic and single beam data. Detector response is in good agreement not just among sampling layers but also among partitions, and the barrel response is already consistent within $6 \%$.

\section{Performance Studies of Calorimeter Clusters}

There are 2 major clusterings in the ATLAS calorimeters [9]. They are traditional combined towers which group cells from all sampling layers of EM and hadronic calorimeters within the same $\Delta \eta \times \Delta \phi$ range (and some merging afterward) and "Topological Clustering" which group neighboring cells with significant energy deposit compared to the expected noise

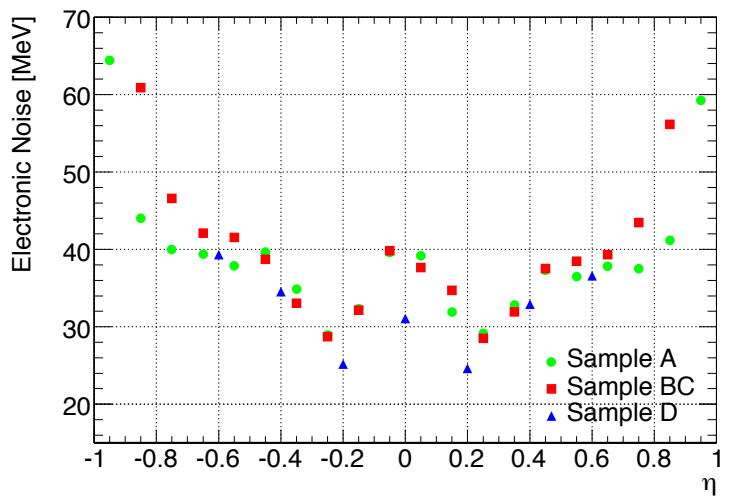

Fig. 13. $\quad \eta$ dependence of electronic noise in the Long-Barrel.

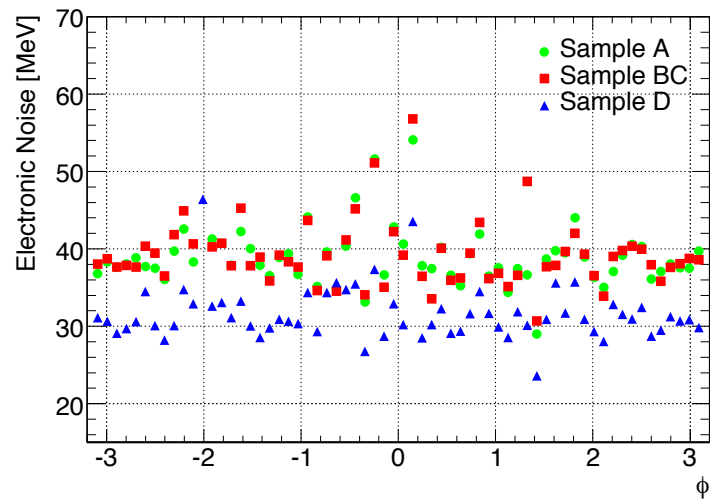

Fig. 14. $\phi$ dependence of electronic noise in LBA.

(see section C.2. for more detail). These clusters are used as inputs for jet reconstruction and Missing Transverse Energy (Missing $\mathrm{E}_{T}$ ) calculation which are important observables for physics measurements. Topological clusters (Topoclusters) are expected to provide better noise suppression compared to the combined towers, and some additional information such as cluster shape and etc. In order to use the Topoclusters, electronic noise must be measured cell by cell.

1) In-situ Noise Measurement: During the cosmic and single beam runs, electronic noise was measured at the cell level. Its dependence on $\eta$ and $\phi$ is shown in Fig. 13 and Fig. 14. In Fig. 13, the cell energy in the same $\eta$ region was averaged over $\phi$ (and vice-versa for Fig. 14). The slight $\eta$ dependence of noise is due to power distribution. The noise was basically uniform per $\phi$ which was expected. Stability of the average noise value in LBA is shown in Fig. 15 for a 3 month period. The stability was within $2 \%$. For clustering and other physics object reconstruction, noise values are provided cell by cell from the ATLAS database. The noise values will be continuously monitored during the physics run, and any significant change will be investigated and the database will be updated.

2) Topological Clustering Algorithm: During the clustering, cells are grouped into "seeds", "neighbors" and "others" in regards to their signal-to-noise ratio $(\mathrm{S} / \mathrm{N})$. In the standard 


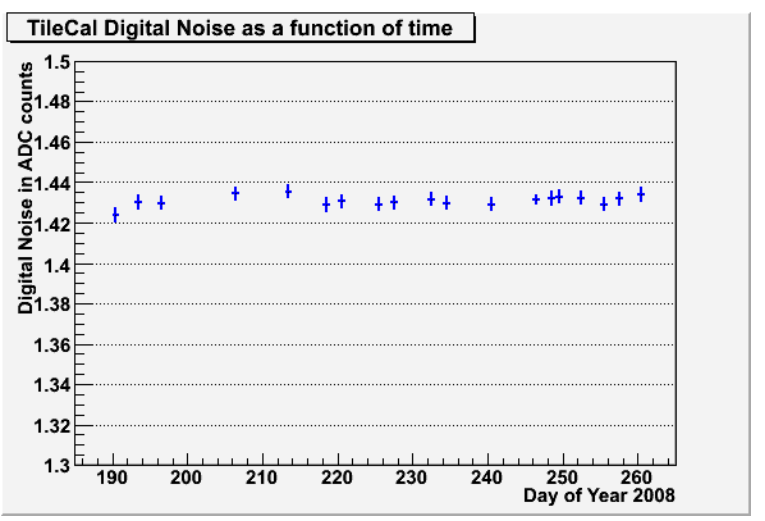

Fig. 15. Stability of the average noise values in LBA over 3 months period.

clustering scheme, cells with $|\mathrm{S} / \mathrm{N}|>4$ serve as "seeds". ${ }^{1}$ Neighboring cells with $|\mathrm{S} / \mathrm{N}|>2$ are defined as "neighbors" and added to the clusters which themselves can behave as new "seeds", expanding the clusters by including their adjacent cells with $|\mathrm{S} / \mathrm{N}|>2$. All the neighboring cells to "neighbors" are defined as "others" and added to the clusters without any threshold.

This algorithm is intended to group cells in 3 dimensions and among all the calorimeters (EM LAr calorimeter, Tile hadronic calorimeter, LAr hadronic and forward calorimeter). Clusters consisting only from TileCal cells were used for commissioning as well.

3) Performance of TileCal Topoclusters: Topoclusters reconstructed only from TileCal cells were used for the commissioning. The energy distribution of TileCal Topoclusters from a cosmic run in November 2007 is shown in Fig. 16. Only LBA and LBC was read out during this run. Since noisy channels form fake clusters, identified problematic channels were removed during the offline reconstruction.

TGC-triggered (TGC: Thin Gap Chamber) and TileCaltriggered events were investigated. TGC is the end-cap muon trigger, so those events rarely have cosmic muons passing the Long-Barrel of TileCal. The TileCal-trigger selects events that have large energy deposit in the top and bottom drawers. Coincidence of top and bottom towers was required, and high purity of cosmic muons were observed in the triggered events. In Fig. 16, TileCal Topoclusters in TGC-triggered were mainly from noise, whereas many of the Topoclusters in TileCal-triggered events were originated from cosmic muon energy deposit. Though the identification of the problematic channels was preliminary, and optimization of threshold for cluster reconstruction ${ }^{2}$ needs further investigation, a clear separation was seen between noise-like clusters and muon energy deposit. This indicates that despite that Topoclusters have good noise suppression, they can tag low energy deposit even from minimum ionizing particle (MIP) in TileCal. Further investigation is ongoing with combined Topoclusters that are formed from all the calorimeters.

\footnotetext{
${ }^{1}$ Threshold is applied to the absolute value to avoid selection bias on positive side when energy sum of clusters is calculated for jets or Missing $E_{T}$. Negative energy originate from fluctuation around the pedestal value.

${ }^{2}$ Current threshold is optimized from test-beam analysis.
}

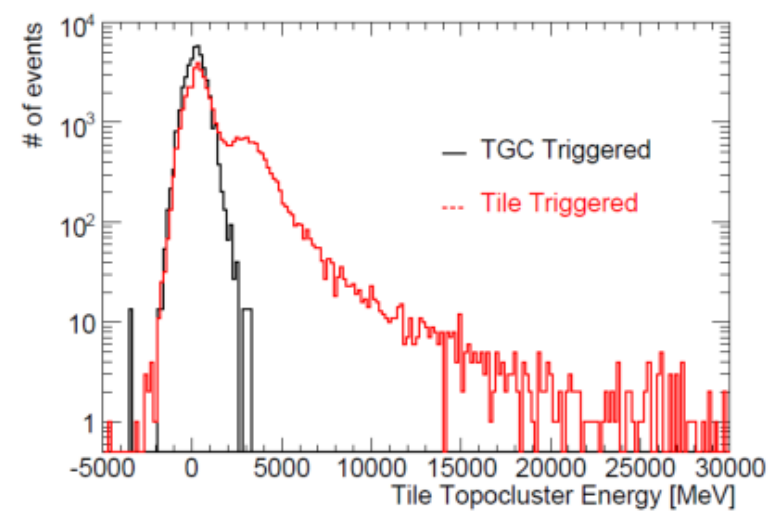

Fig. 16. Energy distribution of Topoclusters reconstructed solely from TileCal. TGC-triggered and TileCal-triggered events in a cosmic run from Nov. 2007 were used.

\section{CONCLUSION}

The current status of TileCal commissioning studies with cosmic and single beam data was shown. Understanding of the ATLAS Tile Calorimeter has greatly improved through the combined cosmic and single beam runs. Validation of timing calibration was done with both types of runs, and good agreement with the laser calibration was observed. With the single beam runs, timing information from all the partition was within 1 bunch crossing, but slight timing discontinuity was observed among different partitions which was due to the different time reference used in each partition. This effect will be taken into account for timing reconstruction. Detector energy response was in good agreement between cosmic and single beam data, and uniformity of the energy response among all sampling layers and partitions was already in good shape (within $6 \%$ in the barrel). Finally, electronic noise measurement was performed at the cell-level, and the measured values were used for the cluster reconstruction. The performance of the Topological Clustering algorithm was investigated, and a clear separation between noise and muon energy deposit was observed from Topoclusters solely reconstructed from TileCal. Investigation is ongoing with combined Topoclusters which leads to jet reconstruction and Missing $\mathrm{E}_{T}$ calculation.

\section{ACKNOWLEDGMENT}

We would like to thank the ATLAS Tile Calorimeter Group for their contributions, especially Francesc Vives, Matteo Volpi, Luca Fiorini, and Ilya Korolkov. H. Okawa would like to thank Richard Teuscher and other experts for many fruitful discussions.

\section{REFERENCES}

[1] J. J. Engelen, "Recent Highlights from the Large Hadron Collider Project," in these proceedings.

[2] G. Aad et al. [ATLAS Collaboration],"The ATLAS Experiment at the CERN Large Hadron Collider," JINST 3 S08003, (2008).

[3] ATLAS Tile Calorimeter Collaboration, "Tile Calorimeter Technical Design Report," CERN/LHCC/96-42, (1996).

[4] M. Bosman et al., "Developments for a scintillator tile sampling hadron calorimeter with "longitudinal" tile configuration," CERN/DRDC/93-3, (1993).

[5] A. Wright, "First 'data' from LHC," Nature Physics, doi:10.1038/nphys005, (2005). 
[6] A. Gibson, "Commissioning of the ATLAS Reconstruction Software with First Data," in these proceedings.

[7] L. Fiorini, I. Korolkov, F. Vives, "Time Calibration of TileCal Modules with Cosmic Muons," ATL-TILECAL-PUB-2008-010, (2008).

[8] J. Maneira, "Commissioning of the ATLAS Offline Software with cosmic rays,' IEEE NSS-MIC '07, October 27-November 3, 2007, Honolulu, USA.

[9] W. Lampl et al., "Calorimeter Clustering Algorithms: Description and Performance," ATL-LARG-PUB-2008-002, (2008). 